\title{
Current Density Calculation of a High Frequency Ion Source
}

\author{
H. Heydari \\ Technical University, EN-2 \\ D-1000 Berlin 10
}

\begin{abstract}
The current density of a high frequency charged particle source is calculated using the Child-Langmuir formula and the quantummechanical transition probability for ionization processes at fixed gas pressure and temperature. The dependence of the current density on the differential ionization coefficient and various geometrical and electromagnetic quantites is taken into account. The fundamental mechanism of the ion source considered consist in the initial natural ionization and in the application of high frequency fields in order to accelerate the electrons and ions against the gas molecules thus yielding considerable ionization. Plasma physical phenomena are not relevant and the calculation agrees well with experimental data for the range considered.
\end{abstract}

\section{Introduction}

We present a theoretical approach concerning the determination of the current density of an jon source, for which the construction scheme is illustrated in Fig. 1.

While applying a high frcquency ficld on the mid-section of the piston neutral atoms will be ionized. In the beginning the ionization is caused mainly through accelerated free electrons. Later, however, also secondary electrons originating from shells will play a stronger role for the ionization. Inside the mid-section of the piston one can consider a highly conductive plasma with only small variations of the potential. Near cathode and anode, however, one expects an ensemble of positiv ions and electrons respectively. As approximation the Child-Langmuir equation [1] describes the correlation between current and voltage inside the high vacuum tube.

$$
I_{-}=\sqrt{\frac{2 e}{m_{e}}} \frac{U_{-}^{3 / 2}}{9 \pi d s^{2}}
$$

The indirect heating of the cathode through collisions with ions as well as the tunneleffect of the neutral gas atom are subject to no considerable contribution of the ionization rate in this apparatus. Also statistical physical effects (i.e. Boltzmann-distribution) are at temperatures around $300^{\circ} K$ irrelevant. Only temperatures greater than

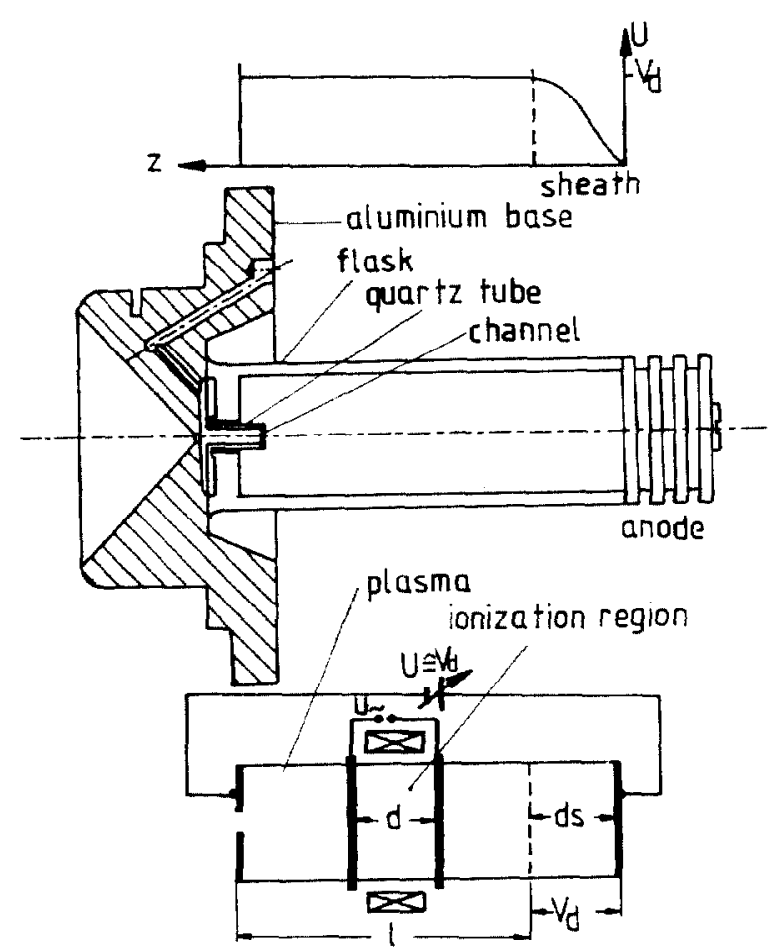

Figure 1: The high frequency ion source plane section

$16 \cdot 10^{4} \mathrm{~K}$ would offer the necessary thermic energy for ionization.

\section{Calculation of the ionization probability}

To calculate the number of produced ions during one second and inside one $\mathrm{cm}^{2}$ we first determine the ionization probability for a collision of a fast electron and a neutral atom (see Fig. 2). In a first approximation the colliding electron will move with a velocity $v_{e}$ along a straight linc, having in $D$ the shortest distance to the nucleus. The transition probability for the transition from state $n$ to state $m$ can be calculated by taking the timedependent perturbation theory into account. With the "Fermi golden rule" 


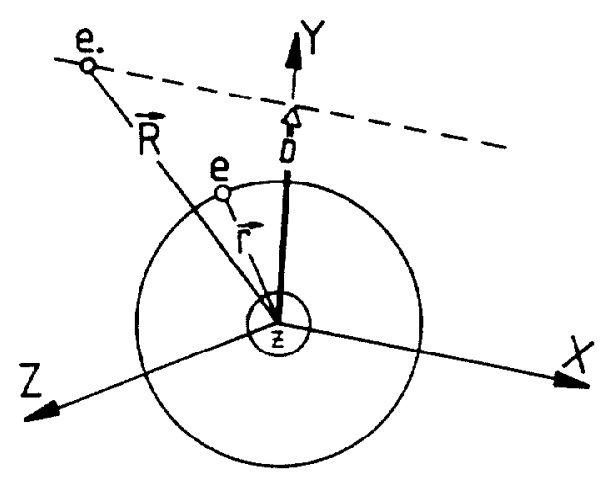

Figure 2: Simplified model to determine the ionization-probability

and the Hamilton operator [2] for the discussed system of atoms colliding with electrons we receive the following expression for the transition probability

$$
U_{n m}(\tau)=-\frac{1}{\hbar^{2}}\left|\int_{\tau}\langle n|w(t)| m\rangle \mathrm{e}^{i \omega_{n m} t} \mathrm{~d} t\right|^{2}
$$

with the perturbation operator

$$
w(t)=(-1) \frac{Z e^{2}}{4 \pi \varepsilon_{0}|\vec{R}-\vec{r}|}
$$

depending on the transition energy

$$
\hbar \omega_{n m}=E_{n}^{0}-E_{m}^{0}
$$

After introducing an effective collision time $t_{s t}=D / v_{e}$ one
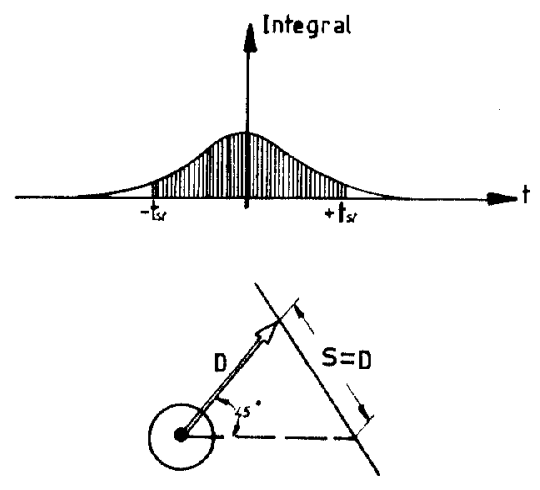

Figure 3: For determination of the distance $D$ and time $t_{s t}$

receives the following equation

$$
\begin{gathered}
U_{n m}(-\infty,+\infty) \approx \frac{1}{\hbar^{2}} Z^{2}\left(\frac{e^{2}}{4 \pi \varepsilon_{0}}\right)^{2} \cdot \frac{4\left|Y_{n m}\right|^{2}}{v_{e}^{2} D^{2}} \\
|\vec{D}| \gg|\vec{r}|=a
\end{gathered}
$$

for an ionization process caused by Coulomb interaction between the colliding electron and electron shell of the investigated atom. $Y_{n m}=\langle n|y| m\rangle$ is the matrix-element of the $y$-component of the position operator.
Here we describe a non-adiabatic case, since otherwise the passing electron would have not excited the atom. To calculate the probability $\mathrm{d} P_{n m}$ to exite an atom from state $n$ to $m$ caused by interaction of many colliding electrons with a current density of

$$
j=\frac{\mathrm{d} N}{\mathrm{~d} t \mathrm{~d} A}
$$

one multiply the transition probability of (5) with the current density of $(6)$ and integrate the result over the area $\mathrm{d} t 2 \pi D \mathrm{~d} D$ between $D=0$ and $D=v_{e} / \omega_{n m}$. We receive the expression

$$
\mathrm{d} P_{n m}=\int_{0}^{v_{e} / \omega_{n m}} \underbrace{\mathrm{d} t}_{\text {time }} \underbrace{2 \pi D \mathrm{~d} D}_{\text {area }} j U_{n m}^{D}(-\infty,+\infty)
$$

The upper integration border $v_{e} / \omega_{n m}$ characterizes a separation line between the non-adiabatic case $\left(D<v_{e} / \omega_{n m}\right)$ and the adiabatic case $\left(D>v_{e} / \omega_{n m}\right)$. Since $j \mathrm{~d} t 2 \pi D \mathrm{~d} D=$ $\mathrm{d} N$ describes the number of collisions inside a ring around the nucleus of a radius $D$ and thickness $\mathrm{d} D$ one receives for the ionization probability per unit time

$$
\begin{aligned}
& \frac{\mathrm{d} P_{n m}}{\mathrm{~d} t}= \\
& =8 \pi j Z^{2} \alpha^{2}\left(\frac{c}{v_{e}}\right)^{2}\left|Y_{n m}\right|^{2} \ln \left(\frac{v_{e}}{\omega_{n m} a}\right) \\
& =8 \pi j \frac{Z^{2} \alpha^{2}}{2} \frac{c^{2}}{W_{e}} \frac{m_{e}}{2}\left|Y_{n m}\right|^{2} \\
& \times\left(\ln W_{e}-2 \ln \left(e V_{i}\right)+2 \ln \hbar-2 \ln a-\ln \frac{m_{e}}{2}\right)
\end{aligned}
$$

$W_{e}$ symbolizes the collision energy and $e V_{i}$ the ionization energy.

\section{Calculation of the number of produced ions}

If one divides the ionization probability per unit time with the area perpendicular to the particle current and considers the number of neutral gasatoms

$$
N_{n_{A}}=\frac{p V}{k T}
$$

following equation describes the number of ionized atoms per unit time and area

$$
\begin{aligned}
& \dot{n}_{+}=\frac{p l}{k T} 4 \pi \frac{I_{-}}{e} \alpha^{2} \frac{2}{W_{e}} \frac{m_{e}}{2} \\
& \times\left|Y_{\text {gas } \rightarrow i o n}\right|^{2}\left\{\frac{W_{e} h^{2}}{\left(e V_{i}\right)^{2} a^{2} \frac{m_{e}}{2}}\right\} .
\end{aligned}
$$


With the velocity of light $c$, finestructure constant $\alpha$, length $!$, ionization energy $e V_{i}$, collision energy $W_{e}$ of electrons with the charge $e$ and the abbreviations

$$
\begin{aligned}
& C_{1}=\frac{4 \pi}{k T} \alpha^{2} \frac{m_{\epsilon}}{2}\left|Y_{\text {gas } \rightarrow \text { ion }}\right|^{2} e V_{i} \\
& C_{2}=\hbar\left(1 / a^{2} \frac{m_{e}}{2} e V_{i}\right)
\end{aligned}
$$

plus the so called differential ionization coefficient $S_{e}$

$$
\left(C_{1} / W_{e} e V_{i}\right) \ln \left\{C_{2}\left(W_{\varepsilon} / e V_{i}\right)\right\}=S_{e}
$$

expression (10) simplifies to

$$
\dot{n}_{+}=\left(I_{-} p / / e\right) S_{e} .
$$

Presently one can find also other expressions for the ionization coefficient $S_{e}=S_{e}\left(W_{e}\right)$ [3]. In our case, however, only equation (12) is relevant, since the collision encrgy is far greater than the ionization energy $e V_{i}$ and quantummechanical treatment of the ionization process is preferred. Still missing is the determination of the collision energy $W_{e}$ of the clcctrons, which extract their kinetic energy from the high frequency field:

$$
E_{\sim}=\frac{U_{\sim}}{d}=\frac{U_{0}}{d} \sin (\omega t)
$$

The motion of an accelerated electron is described by

$$
m_{e} \ddot{x}=e \frac{U_{0}}{d} \sin (\omega t)
$$

with the start conditions $x(0)=0, \dot{x}(0)=v_{e}$, since the electrons posses an initial velocity $v_{e}$ due to the constant electrical field (highvoltage $U_{-}$). Integration of the equation of motion (14) delivers the velocity $\dot{x}$ of the electrons and therefore the collision energy $W_{e}=\left(m_{e} / 2\right) \dot{x}^{2}$ :

$$
\int_{0}^{t} \mathrm{~d} t \ddot{x}(t)=\int_{0}^{t} \frac{e}{m_{e}} \frac{U_{0}}{d} \sin (\omega t) \mathrm{d} t
$$

The time average of the collision energy results to

$$
\begin{gathered}
\bar{w}_{e}=\frac{1}{T} \int_{0}^{T} W_{e} \mathrm{~d} t= \\
=\frac{m_{e}}{2}\left\{v_{e}^{2}+2 v_{e} \frac{e U_{0}}{m_{e} \omega d}+\frac{3}{2}\left(\frac{e U_{0}}{m_{e} \omega d}\right)^{2}\right\} \\
T=\frac{2 \pi}{\omega}
\end{gathered}
$$

\section{Results, comparision with avai- lable data}

One receives a good approximation for the number of produced ions per unit time and area, if the calculation for the ionization coefficient (12) is done with the time average of the collision energy $\bar{w}_{e}(16)$ instead of the individual collision energy $W_{e}$ itself. The result for (13) depends on the voltage $U_{-}$(see Fig. 1), frequency $f=\omega / 2 \pi$ of the high frequency source and geometry of the ion source, so that the following comparision for $S_{e}$ can be presented (Fig. 4).

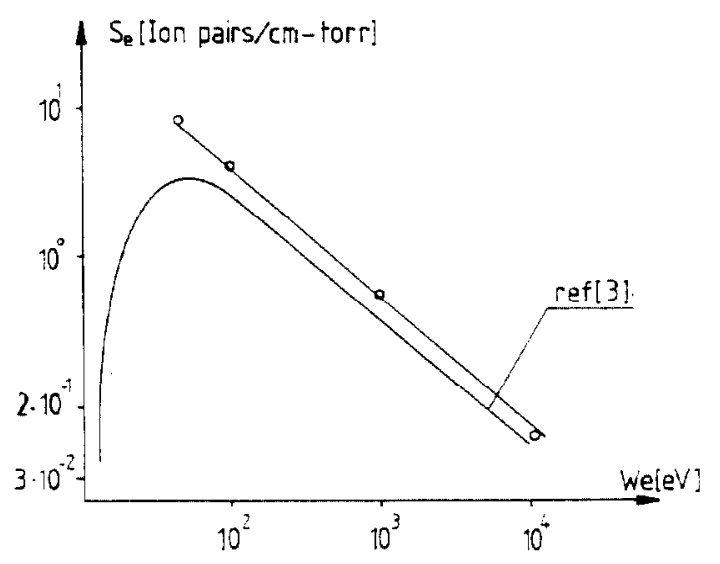

Figure 4: Results from eq.(12) compared with [3]

\section{Conclusion}

Introducing an approach based on a very simple theoretical model for collisional processes of an accelerated electron with a neutral gas atom we presented here an expression describing the number of produced ions per unit time and area in a high frequency ion source. As important parameters the result depends on geometry dimensions, frequency and amplitude of the high frequency source, $U_{-}$-voltage and the gas characteristics. As a further simplification we also employed the linte average of collision energy for our calculations. This approach is justified as experimental results demonstrate, where only a constant ionization current density was registered. We believe that plasmawave effects have only a minor effect in the time average. The in time and position constant magnetic field (see Fig.1), not discussed in this paper, has no influence on the resulting current density, since it proves to only enlarge the effective path length of the colliding electron inside the ionization area.

\section{References}

[1] R.G. Wilson and G.R. Brewer, "Ion Beams with applications to ion implantation", J. Wiley \& Sons, New York, 1973

[2] A. S. Dawydow, "Quantenmechanik", VEB Deutscher Verlag der Wissenschaften, Berlin, 1978

[3] A.V. Eengel and M. Steenbeck, "Elektrische Gasentladungen", Band 2, Berlin, Ileidelberg, New York: Springer, 1934 